\title{
NON EXISTENCE D'UN SPIN DES PHOTONS;
}

\author{
par A. KASTLER.
}

\begin{abstract}
Sommaire. - Le principe de sélection du quantum azimutal et le parallélisme que la mécanique ondulatoire établit entre électrons et photons suggèrent l'idée de l'existence d'un spin des photons correspondant à celui des électrons. On peut supposer une relation entre l'orientation des axes des spins corpusculaires et l'état de polarisation de l'onde associée. L'observation de l'absorption des composantes $\sigma$ dans l'effet Zeeman transversal fournit une expérience cruciale pour ou contre le spin des photons. Cette expérience réalisée une première fois par $R$. Frisch sur la raie de résonance du mercure a donné un résultat négatif (pas de spin du photon). L'auteur a repris l'expérience sur les raies $D$ du sodium et a pu confirmer le résultat de Frisch. Il insiste sur quelques conséquences du résultat négatif trouvé.
\end{abstract}

1. Position du problème. - Le principe de sélection de Rubinowicz et Sommerfeld montre que l'émission ou l'absorption de lumière par un atome s'accompagne d'un saut $\Delta n= \pm 1$ du quantum azimutal. Du point de vue de la " Kugelstrahlung » (émission d'une onde lumineuse sphérique par un seul atome) cette condition apparaît comme une conséquence de la conservation du moment cinétique (moment de la quantité de mouvement) du système atome-éther. Pendant l'émission d'une onde lumineuse l'orbite électronique de l'atome perd une partie de son moment cinétique, et cette partie est transmise à l'onde émise. L'échange de moment cinétique entre l'atome et l'éther est égal à

$$
\Delta I=\frac{h}{2 \pi} \Delta n= \pm \frac{h}{2 \pi}
$$

L'échange inverse a lieu pendant l'absorption ( $\left.{ }^{1}\right)$.

Les considérations précédentes transposées dans la théorie de la "Nadelstrahlung » (émission dirigée de photons) font supposer que dans l'émission lumineuse la variation de moment cinétique de l'atome est transmise au photon individuel éjecté qui restituerait ce moment cinétique à un autre atome dans le processus d’absorption. La règle de sélection attribuerait donc au photon individuel un moment cinétique ou spin $I=\frac{h}{2 \pi}$ qu'on peut représenter par un vecteur ayant la symétrie d'un cylindre tournant.

Des considérations de symétrie nous suggèrent alors l'idée d'établir une correspondance entre l'orientation dans l'espace des vecteurs spin des quanta de lumière et l'état de polarisation de l'onde lumineuse. Dans la lumière naturelle les axes de spin des photons seraient orientés au hasard, dans la lumière polarisée les vecteurs spin seraient tous parallèles entre eux : vecteur $I$ parallèle au rayon lumineux dans la lumière polarisée circulairement,

(1) Sommrzвld, Atomban und Spektrallinien, chap. 3 : Wellentheorie ùnd Quantentheorie. Erhaltùng vom Impulsmoment. Auswahlprinzip und Polarisationsregel. 
vecteur I perpendiculaire au rayon lumineux et confondu avec le vecteur magnétique de l'onde dans la lumière polarisée rectilignement $\left({ }^{1}\right)$.

De même qu'il existe deux sortes de lumières polarisées circulairement, gauche et droite, correspondant à des sens opposés du vecteur $I$, de même nous prévoyons l'existence de deux sortes de lumière polarisée rectilignement $\alpha$ et $\beta$ qui se distinguent par le sens des vecteurs 1 . La lumière rectiligne ordinaire (par exemple celle obtenue à l'aide d'un nicol) serait un mélange de $\alpha$ et $\beta$. Mais nous pourrions obtenir isolément l'un ou l'autre état, par exemple l'état $\alpha$, en diffusant de la lumière circulaire gauche par des atomes isotropes (gaz inertes) et en observant à angle droit la lumière diffusée. Dans cette diffusion l'orientation du vecteur $I$ des photons déviés ne doit en effet pas changer. Si nous éclairions avec de la lumière circulaire droite, nous recueillerions au contraire de la lumière diffusée $\beta\left({ }^{2}\right)$.

Cette conception du " photon tournant » nous est encore suggérée d'une manière plus générale par le parallélisme que la mécanique ondulatoire établit entre photons et électrons et par le dualisme des aspects onde et corpuscule de ces deux ètres physiques. Les analogies nombreuses entre l'électron et le photon (vibration interne, masse, quantité de mouvement) nous amènent en effet à envisager le " photon tournant » comme pendant de l' "électron tournant ». Et dans les deux domaines on est tenté de faire correspondre à l'orientation dans l'espace des vecteurs spin des corpuscules l'état de polarisation de l'onde associée.

Pour les électrons cette correspondance entre spin et polarisation de l'onde électronique a été affirmée déjà de plusieurs côtés depuis que Rupp nous a fait connaître des ondes électroniques polarisées $\left.{ }^{3}\right)$.

Existe-t-il une expérience décisive permettant d'affirmer ou de rejeter cette hypothèse $\mathrm{du}$ 《 spin des photons " et sa relation avec l'état de polarisation de la lumière? Oui, cette expérience consiste à observer l'absorption des composantes $\sigma$ dans l'effet Zeeman transversal.

Plaçons une source de lumière (source émissive $\boldsymbol{A}$ ) dans un champ magnétique $\boldsymbol{H}_{1}$ et observons l'absorption de la lumière qu'elle émet par une source absorbante $B$ identique à $A$ et placée elle aussi dans un deuxième champ magnétique $H_{2}$ identique et parallèle au premier. Dans l'observation longitudinale (parallèlement aux champs) on observe deux ou plusieurs composantes $\sigma$ de polarisation circulaire inverse et l'absorption n'a lieu que si le champ $H_{2}$ est égal à $H_{1}$ et de même sens. L'absorption cesse si l'on renverse le sens du deuxième champ. En effet, dans ce cas les sens de circulation des composantes $\sigma$ de même fréquence ne se correspondent plus dans les deux sources. Cette conclusion se trouve d'ailleurs confirmée par une expérience déjà ancienne de $M$. Cotton qui avait envoyé un spectre continu de lumière circulaire à travers une flamme absorbante placée dans un champ magnétique. Seule la composante $\sigma$ de même sens de circulation absorbe la lumière incidente et apparait comme raie renversée $\left(^{4}\right)$.

Mais pour le problème qui nous préoccupe ici, l'observation perpendiculairement aux champs est d'un intérêt particulier. Dans l'effet Zeeman transversal nous observons à côté d'une ou plusieurs composantes $\omega$ vibrant parallèlement au champ, des composantes $\sigma$ vibrant rectilignement perpendiculairement au champ. Ces vibrations $\sigma$ sont en quelque sorte les vibrations circulaires observées dans l'effet longitudinal, et vues maintenant par la « tranche ». Elles correspondent donc les unes à l'état $\alpha$, les autres à l'état $\beta$.

Lorsque les deux champs $\boldsymbol{H}_{1}$ et $\boldsymbol{H}_{2}$ auront même sens, la source $B$ absorbera évidemment

(1) Une telle hypothèse avait déjà été formulée par Henriot, Bulletin de la Classe des Sciences de l'Ac. Royale de Belgique, t. 15 (1929), p. 81.

(2) Remarquons cependant que la différence entre les deux états $\alpha$ et $\beta$ de lumière rectiligne serait moins profonde que celle qui existe entre des lumières circulaires gauche et droite. La différence entre ces deux dernières est en effet absolue (non superposables) alors que la différence entre $\alpha$ et $\beta$ n'existe que relativement à l'observateur qui recueille la lumière.

(3) E. Rupp. Z. Plyysik, 61 (1930), p. 158. - Fues et Hellmann. Physik Z., 31 (1930), p. 465. - Halperr. Z. Physik, 67 (1931), p. 320 : " Die Polarisation einer Materiewelle aùssert sich im Sinne der Dirac-schen Theorie dùrch die Orientierùng des Spinvektois des Elektrons."

(4) Voir Ollivier, Plyysique génerale, tome 2, p. 318. 
la lumière émise par $A$. Mais que se passera-t-illorsqu'on renversera le sens du champ $H_{2}$ ? La théorie électromagnétique classique veut qu'il y ait encore absorption. Mais si nous admettons l'existence d'un spin des photons, l'absorption ne devra plus se produire pour des champs antiparallèles. En effet, dans ce cas les moments cinétiques des électrons de mème fréquence sont opposés dans les sources émissive et absorbante. L'expérience seule pourra décider entre les deux points de vue et son résultat permettra de se prononcer pour ou contre l'hypothèse d'un spin des photons $\left(^{1}\right)$.

Cette expérience a été réalisée une première fois par $\mathbf{R}$. Frisch sur la raie de résonance du mercure et a fourni un résultat négatif (pas de changement dans l'absorption lorsqu'on renverse le champ $\boldsymbol{H}_{2}$ ). J'ai tenu à reprendre l'expérience en variantles conditions : champs magnétiques de 3 à 7000 gauss, raies $D$ du sodium, observation à travers un nicol pour éliminer les composantes $\omega$.

2. Partie expérimentale : Absorption des composantes $\sigma$ dans l'effet Zeeman transversal des raies $D$ du sodium. - L'écart Zeeman normal correspond à $\Delta \nu_{0}=0,047 \mathrm{~cm}^{-1}$ pour un champ $H=1000$ gauss.

L'écart entre les 2 raies $D_{1}=5896 \AA$ et $D_{2}=5890 \AA$ est $\Delta v=17,3 \mathrm{~cm}^{-1}$. Dans les champs accessibles l'effet Zeeman anormal n'est donc pas troublé par l'effet PaschenBack.

L'effet Zeeman anormal des raies $D$ se fait d'après le schéma suivant :

$$
D_{1} \frac{(2), 4}{3}, \quad D_{2} \frac{(1), 3, !)}{3} \text {. }
$$

L'écart des composantes $\sigma$ est donc supérieur ou égal à l'écart normal. Pour obtenir une séparation convenable des composantes Zeeman, il faut employer des champs magnétiques assez forts pour que cet écart soit supérieur à l'épaisseur des raies. La largeur des raies d'une flamme étant de l'ordre de $0,2 \mathrm{~cm}^{-1}$, il est bon d'opérer duns des champs de 6 à 8000 gauss lorsque les sources $\mathrm{A}$ et $\mathrm{B}$ sont des flammes de sodium.

Lorsqu'on utilise la résonance optique, on peut obtenir un résultat net dans des champs beaucoup plus faibles, à cause de la finesse des raies de résonance. La largeur des raies de résonance est due uniquement à l'effet Doppler-Fizeau et s'évalue ainsi à $0,07 \mathrm{~cm}^{-1}$. Il suffit donc d'opérer dans des champs de 3000 gauss. Il n'est pas bon d'aller au-delà, la flamme excitatrice ne couvrant plus dans des champs trop forts.

J'ai eu à ma disposition 2 électro-aimants identiques type Weiss, petit modèle, me donnant facilement les champs nécessaires. Leurs caractéristiques ont été établies par un fluxmètre. Une mesure absolue des champs a été faite avec une balance de Cotton. Les pièces polaires étaient des cylindres plats de $6 \mathrm{~cm}$ de diamètre. Les entrefers avaient une épaisseur de 2 à $3,6 \mathrm{~cm}$. Les deux champs étaient ainsi suffisamment uniformes. Les électroaimants étaient placés côte à côte ; les centres des entrefers étant distants d'une trentaine de centimètres, les deux champs ne se troublaient pas mutuellement. Je n'ai fait que des observations sur l'effet Zeeman transversal.

Les expériences ont été effectuées de deux manières différentes:

a) Avec des flammes de sodium. - La source A était obtenue en colorant faiblement la flamme d'un chalumeau gaz-oxygène par une baguette de pyrex amenée au contact de la flamme. La source B était obtenue en volatilisant du bromure de sodium dans la flamme bleue d'un bec Bunsen. Une petite lentille donnait une image réelle de la flamme A à l'empla-

(1) Les idées précédentes avaient été développées par l'auteur au début de l'année 1930 et communiquées par lui, en février 1930, à M. Eugène Bloch, professeur à la Sorbonne qui l'engagea à faire l'expérience. Sa réalisation ayant été retardée par des difficultés matérielles, l'auteur a été devancé dans cette voie par R. Frisch, Zur Drehimpulsbilanz bei Lichtemissionsvorgangen, Z. Physik, 61 (1930), p. 626. Cet auteur, partant d'une idée analogue, avait conçu et réalisé la même expérience. La priorité de publication du sujet appartient donc à M. Frisch. 
cement de la flamme B. La flamme B et cette image de A s'observaient à travers un prisme de Nicol à champ normal qui ne laissait passer que les vibrations perpendiculaires aux champs magnétiques (composantes 5 ). En réglant convenałlement l'intensité des flammes, on voit se détacher nettement le bord noir absorbant de la flamme B sur le fond clair de l'image de A. La méthode consiste ensuite à observer l'aspect de ce bord lorsqu'on modifie les champs magnétiques (méthode de Cotton).

En créant le premier champ, $\boldsymbol{H}_{1}$, on voit disparaătre presqu'entièrement la bande noire qui borde la flamme B. Mais cette bande noire réapparaît lorsque l'on excite à son tour le deuxième électroaimant, et ceci quelque soit le sens du champ $\mathrm{H}_{2}$

J'ai utilisé des ehamps de 7200 gauss.

b) En utilisant la résonance optique. - En se servant de la résonance optique de la vapeur de sodium, on peut obtenir un résultat très net dans des champs plus faibles. J'ai opéré dans ce cas avec succès dans des champs de 3000 gauss. La source émissive A était constituée par un petit tube scellë en verre sībor contenant du sodium métallique purifié par distilllation dans le vide. Ce tube était éclairẻ par une flamme de sodium intense obtenue en introduisant une baguette de pyrex dans un chalumeau gaz-oxygène.

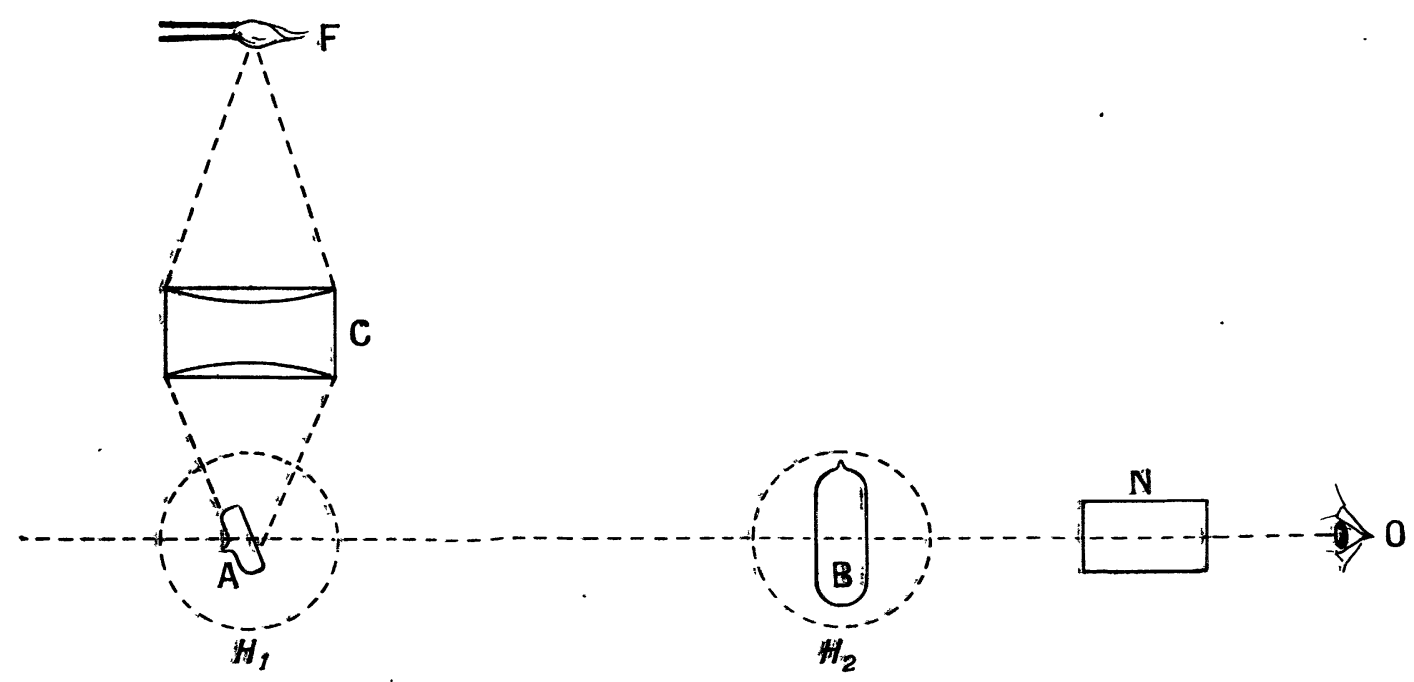

Fig. 1 .

F, flamme de sodium

C, Contenseur

N, Aicol.

La lumière du chalumeau était conoentrée par un condenseur sur le tabe A placé dans l'entrefer $\boldsymbol{H}_{1}$. Il suffisait de chauffer ce tube doucement dans la flamme d'wn bec Bunsen pour Lui faire émettre une limière de résonance persistant pendant plusieurs minutes et s'afifaiblissant lentement pendant le refroidissement du tube. Cette source de résonanoe $A$ était observée à travers un nicol et un deuxième tube scellé $\mathrm{B}$ plus large, contenant également du sodium pur et plaoé dans l'entréer $\boldsymbol{H}_{2}$ du deuxième électro-aimant.

Les deax tubes étaient chauffés simultanément, puis l'on procédait à l'observation. En l'absence de champs magnétiques $\mathrm{A}$ était invisible à travers $\mathrm{B}$. La lumière de résonance émise par A était donc intégralement absorbée par B. En établissant alors le champ $H_{1}$, on voyait le tube A s'illuminer. 
L'établissement du deuxième champ $H_{2}$ éteignait de nouveau le tube $\mathrm{A}$, et rétablissait ainsi l'absorption et ceci quel que soit le sens du champ $\left.\mathrm{H}_{\mathbf{2}}{ }^{1}\right)$.

3. Conséquences théoriques. - Ces expériences confirment donc entièrement le résultat de Frisch : l'absorption des composantes $\sigma$ dans l'effet Zeeman transversal est indépendante du sens relatif des champs magnétiques $\mathrm{H}_{1}$ et $\mathrm{H}_{2}$. La dissymétrie qui se manifeste dans l'effet Zeeman longitudinal lorsqu'on renverse l'un des champs ne se révèle pas dans l'effet transversal.

Ce résultat qui est en accord avec la théorie électromagnétique classique condamne l'hypothèse du moment cinétique des photons. Frisch montre dans son mémoire, cité plus haut, comment on peut essayer de concilier ce résultat avec le principe de conservation du moment cinétique dans l'émission et l'absorption de la lumière par un atome. Je voudrais insister ici sur les conséquences de ce résultat négatif pour la structure même de la lumière.

L'analogie entre l'électron et le photon, mise en lumière par L. de Broglie, ne doit pas être exagérée. Le parallélisme qui existe entre ces deux êtres physiques (masse, quantité de mouvement, vibration interne régie par le postulat universel $E=h v=m c^{2}$ ) ne doit pas nous faire oublier les différences irréductibles qui existent entre les deux genres de corpuscules. A côté des propriétés communes aux deux corpuscules l'électron seul possède en outre une charge électrique, un moment cinétique et un moment magnétique.

Ces trois qualités supplémentaires de l'électron coexistent toujours et ne sont que trois manifestations d'une même chose. Il serait artificiel et sans signification physique de séparer les notions de charge électrique, de moment cinétique et de moment magnétique. Le photon par contre semble dépourvu de cette triple propriété. Fréquence, masse, quantité de mouvement sont ses seuls attributs.

Nous venons de voir que nous sommes obligés d'abandonner l'idée du spin des photons. Il faut donc renoncer également à la correspondance entre l'orientation des vecteurs spin et l'état de polarisation de l'onde lumineuse. Il ne reste guère possible de faire correspondre une qualité d'un corpuscule individuel à la notion ondulatoire de polarisation .

L'interprétation quantique de la polarisation doit être recherchée sans doute dans des considérations statistiques. Mais dans ce cas la correspondance entre spin et polarisation, pourtant si séduisante, devient également bien problématique pour les ondes électroniques.

L'expérience suivante serait intéressante à tenter à ce sujet :

Un jet d'électrons pénètre, perpendiculairement aux lignes de force. dans un champ magnétique homogène, où il est dévié, à angle droit par exempjle. En traversant le champ les électrons subissent la quantification dans l'espace. Dans le jet dévié les axes de spin sont donc tous orientés parallèlement au champ. L'onde électronique est-elle alors polarisée? L'étude de sa réflexion sur une feuille d'or, en fonction de l'azimut, pourrait nous apporter la réponse.

(') En observant le tube A directement, on constate d'ailleurs, en donnant le champ $\boldsymbol{H}_{1}$, une modification de la lumière de résonance, qui s'explique facilement par la distribution d'intensité dans les raies de la flamme excitatrice. Lorsque le pyrex effleure à peine la flamme, la raie excitatrice est normale et a son maximum d'intensité au centre. Dans ce cas l'établissement du champ assombrit la résonance.

Si au contraire le pyrex est introduit au milieu de la flamme. qui contient alors beaucoup de sodium en couche épaisse, l'auto-absorption de la flamme produit une raie excitatrice renversée. La production du champ (3000 gauss) renforce alors la lumière de résonance.

Dans des champs de 7000 gauss la résonance s'éteint toujours complètement. L'étude de la résonance optique dans un champ magnétique constitue donc un moyen commode pour étudier sans spectroscope la largeur et la distribution d'intensité d'une raie excitatrice. Il serait facile de rendre la méthode quantitative en étalonnantle champ magnétique et en mesurant photométriquement l'intensité de la lumière réémise par résonance. 
La partie expérimentale de ce travail a été effectuée au laboratoire de physique de l'Ecole Normale Supérieure à Paris. Je tiens à exprimer ma reconnaissance à MM. Eugène et Léon Bloch qui ont bien voulu s'intéresser à ce travail. Je remercie mon ami F. Esclangon qui m'a aidé à monter les expériences. Je dois également des remercîments à M. Guinchant, professeur à la Faculté des Sciences de Bordeaux, qui a mis à ma disposition, pour des essais préliminaires, l'électroaimant Weiss de son laboratoire. 